\title{
Stellar dynamics in young clusters: the formation of massive runaways and very massive runaway mergers
}

\author{
D. Vanbeveren ${ }^{1,2}$, H. Belkus ${ }^{1}$, J. Van Bever ${ }^{3}$, N. \\ Mennekens ${ }^{1}$
}

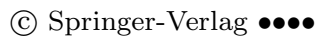

\begin{abstract}
In the present paper we combine an N-body code that simulates the dynamics of young dense stellar systems with a massive star evolution handler that accounts in a realistic way for the effects of stellar wind mass loss. We discuss two topics:

1. The formation and the evolution of very massive stars (with a mass $>120 M_{\odot}$ ) is followed in detail. These very massive stars are formed in the cluster core as a consequence of the successive (physical) collison of 10-20 most massive stars of the cluster (the process is known as runaway merging). The further evolution is governed by stellar wind mass loss during core hydrogen burning and during core helium burning (the WR phase of very massive stars). Our simulations reveal that as a consequence of runaway merging in clusters with solar and supersolar values, massive black holes can be formed but with a maximum mass $\approx 70 M_{\odot}$. In small metallicity clusters however, it cannot be excluded that the runaway merging process is responsible for pair instability supernovae or for the formation of intermediate mass black holes with a mass of several $100 M_{\odot}$.

2. Massive runaways can be formed via the supernova explosion of one of the components in a binary (the Blaauw scenario) or via dynamical interaction of a single star and a binary or between two binaries in a star cluster. We explore the possibility that the most massive runaways (e.g., $\zeta$ Pup, $\lambda$ Cep, $\mathrm{BD}+43^{\circ} 3654$ ) are the product of the collision and merger of 2 or 3 massive stars.
\end{abstract}

D. Vanbeveren, H. Belkus, J. Van Bever, N. Mennekens

${ }^{1}$ Astrophysical Institute, Vrije Universiteit Brussel, Brussels, Belgium,

dvbevere@vub.ac.be, hbelkus@vub.ac.be,nmenneke@vub.ac.be

${ }^{2}$ Groep T, Association K.U.Leuven, Leuven, Belgium

${ }^{3}$ Institute of Computational Astrophysics, St.-Mary's University, Halifax, Canada,

vanbever@penguin.stmarys.ca
Keywords Cluster stellar dynamics, massive star evolution, massive star winds

\section{Introduction}

The temporal evolution of the population of different types of massive stars in starbursts has been the subject of numerous research topics in the last two decades. We can distinguish studies dealing with OBtype stars, Wolf-Rayet (WR) type stars and compact objects. Some of them have only been concerned with single stars (e.g. Arnault et al. 1989; Maeder 1991; Mas-Hesse \& Kunth 1991; Cervino \& Mas-Hesse 1994; Meynet 1995; Leitherer et al. 1999). The effects of close binaries were investigated by Dalton \& Sarazin (1995); Schaerer \& Vacca (1998); Pols et al. (1991); Van Bever \& Vanbeveren (1997, 2000, 2003); Vanbeveren et al. (1997, 1998a, b, c); Belczynski et al. (2002). However, none of the papers listed above account for the effects of stellar dynamics, although young massive starbursts may be very dense. Attempts to include dynamics in order to follow the early evolution of massive starbursts have been presented by Portegies Zwart et al. (1999), Ebisuzaki et al. (2001); Portegies Zwart \& McMillan (2002); Gürkan et al. (2004); Freitag et al. (2006). These papers investigate the starburst conditions to initiate a runaway collision (runaway merger), which may lead to the formation of a very massive object at the centre of the cluster, and the possible formation of an intermediate mass black hole (IMBH). IMBHs and their formation became a hot topic soon after the ROSAT and Einstein X-ray surveys of galaxies, when it was realised that the high-luminosity sources (with luminosity up to $\sim 10^{42} \mathrm{erg} / \mathrm{s}$ ) were linked to regions of intense star formation activity, starbursts. Despite the limited sensitivity and spatial resolution of both telescopes, the images of nearby galaxies suggested the 
presence of highly super-Eddington stellar-mass sources with luminosities as high as $\sim 10^{40} \mathrm{erg} / \mathrm{s}$. The X-ray telescope on board of Chandra confirmed the existence of these sources, but it also revealed the existence of individual sources with a luminosity $\sim 10^{41}-10^{42} \mathrm{erg} / \mathrm{s}$ (Ptak \& Colbert, 2004 for a review of galaxies with Ultra Luminous X-ray sources, ULXs). Many models to explain ULXs have been proposed in literature (e.g., Fabbiano, 1989; Colbert \& Mushotsky, 1999; Perna \& Stella, 2004, and references therein) and IMBHs is one of them. MGG 11 is a young dense star cluster $\sim 200 \mathrm{pc}$ from the centre of the starburst galaxy M 82, whose parameters have been studied by McCrady et al. (2003). A ULX associated with the cluster, the runaway collision process and formation of an IMBH in order to explain this source was promoted by Portegies Zwart et al. (2004). The question whether or not an IMBH is needed in order to explain ULXs was addressed in detail by Soria (2007), who concluded that most of them can be explained with a $50-100 M_{\odot} \mathrm{BH}$ accreting mass at super-Eddington rates.

At the center of the Galactic bulge lies a supermassive black hole $\left(\mathrm{SMBH}\right.$ with a mass $\sim 3-4.10^{6} M_{\odot}$, Ghez et al., 1998, 2000). Several young ( $\leq 10 \mathrm{Myr})$ dense star clusters were observed within $\sim 100 \mathrm{pc}$ of this SMBH (Arches, Figer et al., 2002; Quintuplet, Figer et al., 1999; IRS 13E, Maillard et al., 2004; IRS 16SW, Lu et al., 2005). The formation of IMBHs in bulge clusters of this type has been investigated by Portegies Zwart et al. (2006). The authors concluded that IMBHs that are formed as a consequence of core collapse accompanied by runaway star collisions in dense clusters with the properties of bulge clusters, may be the building blocks of SMBHs, a model that was originally proposed by Ebisuzaki et al. (2001).

The runaway merger process in dense stellar systems as a model to explain IMBHs contains two major uncertainties: first, the core collapse and the formation of a runaway merger can be considered as facts, but it is as yet unclear whether or not a very massive object like this will ever become a star, and, secondly, when this object becomes a very massive star, what the effect of stellar wind mass loss is on its evolution and on the final mass the moment that the star collapses. The evolution of very massive stars has been discussed recently by Belkus et al. (2007) and it was concluded that stellar wind mass losses during core hydrogen burning and during core helium burning are very important. A convenient evolutionary recipe for such very massive stars was presented, a recipe that can easily be implemented in a N-body dynamical code. In the present paper we first investigate the formation and evolution of very massive stars in a dense cluster environment. In section 2 we present our N-body code, whereas section 3 summarizes the massive star evolution handler. In section 4 we present simulations of the dynamical evolution of a cluster combined with the massive and very massive evolution handler, and we discuss the results in relation to the cluster MGG 11.

Massive star runaways are defined as massive stars with a peculiar space velocity $\geq 30-40 \mathrm{~km} / \mathrm{s}$. At least $10 \%$ of the O-type stars are classified as runaways (Gies \& Bolton, 1986). They can form either by the dynamical ejection from a cluster due to single starbinary or binary-binary interactions (Poveda et al., 1967; Leonard \& Duncan, 1988, 1990), or by the explosion as a supernova (SN) of a member of a close binary (Blaauw, 1961). A word of caution: it is not because a runaway is observed close to a dense cluster that one should favor the dynamical formation mechanism. If most of the stars are formed in clusters, also binary-SN formed runaways will come out of a cluster. On the other hand, it is not because there is no O-type cluster observed in the neighbourhood of an O-type runaway that one should favor the binary-SN mechanism. Dynamically formed runaways are in many cases rejuvenated collision products (stellar mergers of 2-3 stars, see section 5) and the other O-type stars of the parent cluster of the runaway may have disappeared already.

$\zeta$ Pup, $\lambda$ Cep and $\mathrm{BD}+43^{\circ} 3654$ are 3 most massive runaways with a runaway velocity between $40 \mathrm{~km} / \mathrm{s}$ and $70 \mathrm{~km} / \mathrm{s}$ (Vanbeveren et al., 1998b, c; Hoogerwerf et al., 2001; Comeron \& Pasquali, 2007). In section 5 we explore the dynamical ejection process in order to explain their properties.

\section{The dynamical N-body code for young dense stellar systems}

A full description of our N-body code will be presented elsewhere (Van Bever et al., 2008). Summarizing, our code (written in $\mathrm{C}++$ ) contains three main components. There is the standard scheme for integrating single stars and the centers of mass of stellar hierarchies (e.g., binaries), for which we use the fourth order Hermite scheme described by Makino \& Aarseth (1992). The treatment of compact subsystems requires special techniques that deal with the extremely small interparticle distances that occur in these cases. We distinguish between two-body motion, which is handled by the Kustaanheimo-Stiefel regularisation technique, and more complex encounters between more than 2 stars. The latter is treated by the so-called chain regularization (Mikkola \& Aarseth, 1993, and references therein), which allows the accurate integration of a compact subsystem with arbitrary membership. 
The code generates a zero age massive single star population according to a predefined IMF and a predefined spatial cluster distribution. We use a King model with various concentrations, parametrized by the dimensionless central potential, $W_{0}$. We do not account for the presence of a primordial binary population in the cluster simulations of the present paper.

\section{The massive single star evolution handler}

Our preferred evolutionary model for massive single stars has been described in a number of papers (Vanbeveren et al. 1998a, b, c; Van Bever \& Vanbeveren, 1997, 2000, 2003 and references therein). It is summarized here together with a few updates.

1. Stars with an initial mass larger than $40 M_{\odot}$ evolve according to the LBV scenario as it was introduced by Vanbeveren (1991). Summarizing: due to the fact that no yellow or red supergiants (YSG and RSG) are observed brighter than $\mathrm{M}_{b o l}=-9.5$, we use as a working hypothesis in our evolutionary computations that the $\dot{M}$ during the $L B V$ phase of a star with $M_{b o l} \leq-9.5$ must be sufficiently large to suppress a large expansion, hence to prohibit the redward evolution in the HR-diagram. When this criterion is implemented into a stellar evolutionary code, the code calculates the mass loss rates that are needed at any time in order to prohibit the redward evolution. Since we do not observe RSGs with $\mathrm{M}_{b o l} \leq$ -9.5 in the LMC or SMC either, we consider this as evidence that the LBV scenario is independent from the initial metallicity.

2. The RSG evolutionary phase of massive single stars with an initial mass $<40 M_{\odot}$ is computed in most of the present single star evolutionary codes by using the de Jager et al. (1988) stellar wind mass loss rate

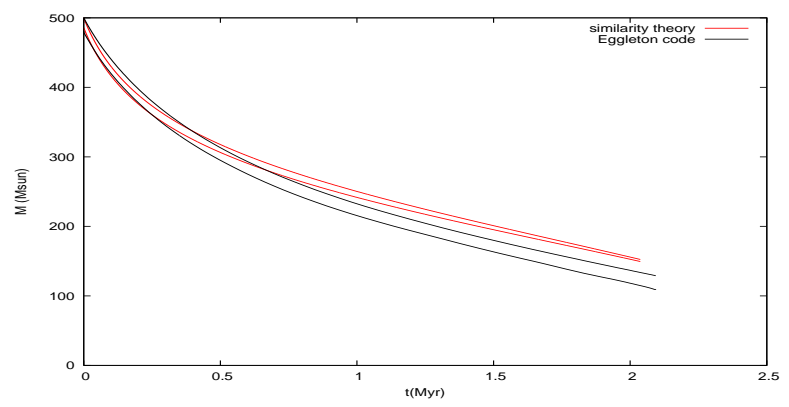

Fig. 1 The temporal evolution during core hydrogen burning of the total mass and of the core mass of a $500 M_{\odot}$ star calculated with the Eggleton stellar evolutionary code (black color) and with the very massive star evolutionary recipe discussed by Belkus et al. (2007) (red color). formalism (e.g., Meynet \& Maeder, 2003; Eldridge \& Vink, 2006). Vanbeveren (1995) and Vanbeveren et al. (2007) illustrated that an update may be necessary, that affects the evolution of single stars with an initial mass between $20 M_{\odot}$ and $40 M_{\odot}$. Our evolutionary handler accounts for this update.

3. Since 1998 we use core helium burning WR mass loss rates in our evolutionary code, which correspond to empirical rates determined by accounting for the effects of clumping. The WR rates are assumed to be Z-dependent (Vanbeveren et al., 1998b, c; Van Bever \& Vanbeveren, 2003). Notice that the WRmass loss rate formalism critically affects the presupernova mass of a massive star, thus also the final fate (neutron star or black hole) and, in case of a black hole, the mass of this compact object.

4. Realistic dynamical simulations of young dense systems of massive stars reveal the existence of what can be considered as one of the most spectacular events in astrophysics: the gravitational encounter of two objects (single star-single star), (single starbinary) or (binary-binary) resulting in many cases in a physical collision of two stars. A collision of two massive stars in dense stellar environments may initiate a chain reaction where the same collision object merges with other massive stars: the term runaway merger is used. In many cases, this runaway merger may become as massive as $1000 M_{\odot}$ or even larger (Portugies Zwart et al., 2006, and references therein, see also section 4) and to investigate the consequences of this process it is indispensable to know how such an object further evolves. Using 3D smoothed-particle-hydrodynamics (SPH) Suzuki et al. (2007) simulated the collision and merging of 2 massive stars. The evolution during merging depends on the mass ratio of the two colliding stars, but after the thermal adjustment (Kelvin-Helmholtz contraction) the merger is nearly homogenized. In our N-body simulations we therefore assume that massive collision objects are homogenized and become ZAMS stars (with the appropriate chemical composition) on a timescale which is short compared to the stellar evolutionary timescale. Obviously, the evolution of this new massive star will be critically affected by stellar wind mass loss.

INTERMEZZO: Suzuki et al. (2007) simulated the collision of a star with a mass $=88 M_{\odot}$ with a star with the same mass and one with a mass $=28 M_{\odot}$. The merging process is very short (of the order of days) and, interestingly, during the merging the collision object loses $\sim 10 M_{\odot}$. It is tempting to link this collision and merging process to the $\eta$ Car out- 
burst in the 19th century. Note that a collision between a single star and a binary may also explain the observed anomalous eccentricity of the $\eta$ Car binary.

With our evolutionary library of massive single stars, it is straightforward to estimate the evolution of a merger with a mass smaller than $120 M_{\odot}$, given its chemical composition after homogenization. However, what about mergers with a mass larger than $120 M_{\odot}$, up to $1000 M_{\odot}$ (very massive stars $=$ VMSs $)$ ? The evolution of VMSs has been studied by Belkus et al. (2007) where it was demonstrated that stellar wind mass loss plays a crucial role. At solar metallicity and larger, VMSs lose most of their mass on a timescale of the order of 2 million years and end their life as a black hole with a mass less than $75 M_{\odot}$. We extended these calculations for stars with an initial mass up to $3000 M_{\odot}$. Stars with a larger mass have a larger luminosity and thus a larger stellar wind mass loss. Our computations reveal that a $3000 M_{\odot}$ star has a mass loss of the order of $10^{-2} M_{\odot} / \mathrm{yr}$. As a consequence, the final mass at the end of core helium burning is less than $\sim 75 M_{\odot}$ as well. At $\mathrm{Z}=0.001$ (and smaller), the final mass may be a factor 2-3 larger and the formation of an IMBH with a mass of a few hundred $M_{\odot}$ is a possibility. We presented a convenient evolution recipe for VMSs that can easily be implemented in a dynamical-population code. To illustrate the validity of our recipe, figure $1 \mathrm{com}-$ pares evolutionary results of a galactic $500 M_{\odot}$ VMS calculated with the Eggleton code with the evolution predicted by the recipe. As can be noticed, the overall evolutionary results that are important in order to follow the evolution of this object in a dense cluster are very similar.

\section{The formation and evolution of runaway mergers in dense stellar systems}

We simulated the early evolution of a dense cluster core containing 3000 massive single stars (with a mass between $10 M_{\odot}$ and $120 M_{\odot}$ satisfying the Salpeter initial mass function) distributed according to a King model $($ Wo $=9)$. We adopt a half-mass radius $=0.5 \mathrm{pc}$. In figure 2 we show the growth in mass of the collision runaway star with time. Our choice of the half mass radius was motivated by the fact that, with the stellar wind mass loss formalism as the one used by Portegies Zwart et al. (2004, 2006), we confirm the mass evolution of the runaway merger and the possible formation of an IMBH with a mass as large as $1000 M_{\odot}$. However, with a more realistic mass loss rate formalism, the figure illustrates that if a dense cluster has solar or supersolar metallicity the formation of an IMBH is rather unlikely.
The BHs in our simulation have a mass not larger than $75 M_{\odot}$ (see also Belkus et al., 2007). Notice that this may be sufficient in order to explain the presence of a ULX in MGG 11, provided that we accept the X-ray formation scenario of Soria (2007).

In order to investigate the effect of the metallicity on the formation and evolution of runaway mergers (through the effect of $\mathrm{Z}$ on the stellar wind mass loss), we followed the dynamical evolution of the same cluster as the one discussed above, but for $\mathrm{Z}=0.001$. The results are shown in figure 2 as well. The final mass is of the order of $200 M_{\odot}$ and we conclude that the formation of an IMBH is possible in dense clusters with small Z. When small Z globular clusters are preceded by a massive supercluster phase, it can thus not be excluded that during this early phase an IMBH formed.

Notice that pair instability supernovae are expected to happen when the final mass after core helium burning is between $\sim 65 M_{\odot}$ and $\sim 130 M_{\odot}$ (Heger \& Woosley, 2002). The results which are depicted in figure 2 illustrate that in dense clusters with subsolar metallicity pair instability supernovae may happen.

More details of the N-body simulations discussed above are given in Belkus et al. (2008).

\section{The formation of massive runaways}

$\zeta$ Pup, $\lambda$ Cep and $\mathrm{BD}+43^{\circ} 3654$ are 3 massive runaways with a runaway velocity between $40 \mathrm{~km} / \mathrm{s}$ and $70 \mathrm{~km} / \mathrm{s}$. Their location in the HR diagram suggests that they belong to the most massive star sample of the solar neighborhood (Vanbeveren et al., 1998b, c; Hoogerwerf et al., 2001; Comeron \& Pasquali, 2007). Runaways can be formed by the binary-SN scenario (Blaauw, 1961) where the original massive primary (the mass loser when the Roche lobe overflow process happens) explodes and eventually disrupts the binary, leaving a neutron star remnant and a runaway secondary (the mass gainer when the Roche lobe overflow happens). Such a scenario for $\zeta$ Pup was presented by Vanbeveren et al. (1998b, c). To explain the significant surface helium enrichment of the star, its rapid rotation and its runaway velocity $(=70 \mathrm{~km} / \mathrm{s})$, the mass transfer phase and the accretion process must be accompanied by spinning-up and quasi-homogenization of the mass gainer (the full mixing model as it was introduced by Vanbeveren and De Loore, 1994) whereas the overall evolution should have resulted in a pre-SN binary with a period of the order of 4 days. The latter requires some fine-tunning.

To illustrate that the dynamical ejection mechanism is a very valuable alternative, the FEWBODY software 


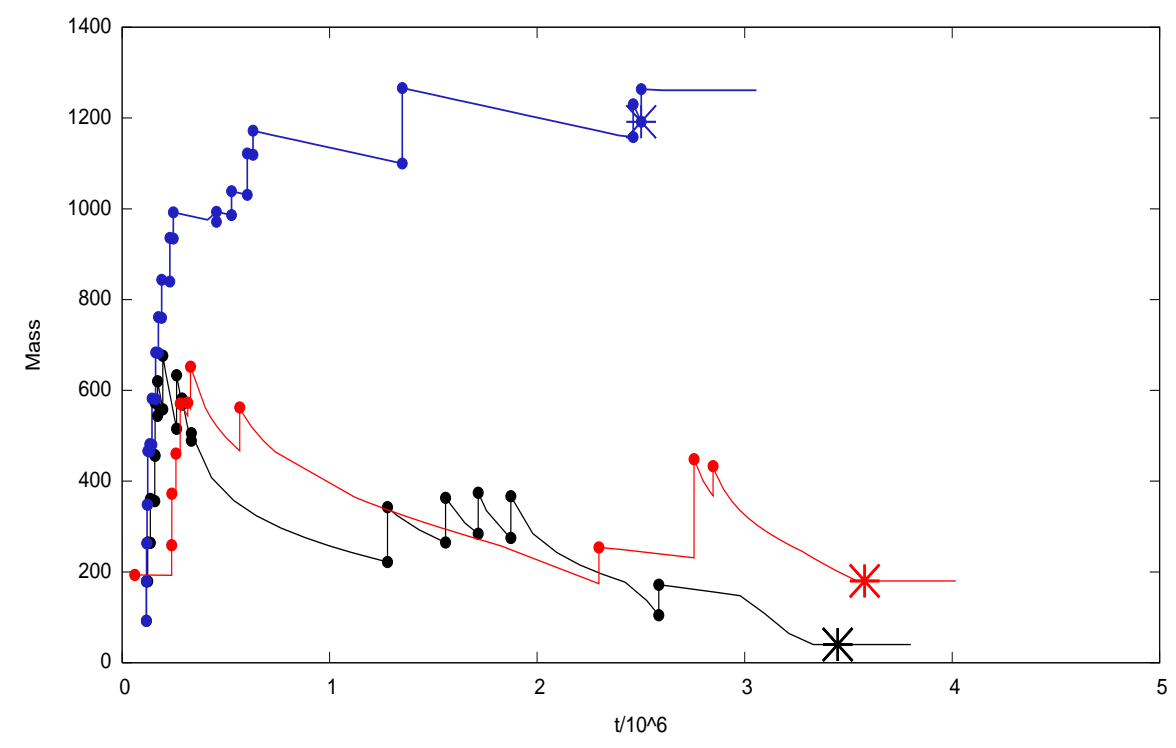

Fig. 2 The effect of stellar wind mass loss on the temporal evolution of the runaway merger during core hydrogen and core helium burning. The blue curve corresponds to a very massive star stellar wind mass loss rate formalism as the one used by Portegies Zwart et al., the black illustrates the evolution with the formalism discussed in Belkus et al. (2007) and metallicity $\mathrm{Z}=0.02$; the red curve is similar as the black one but for $\mathrm{Z}=0.001$.

of Fregeau et al. (2004) was used to reproduce the observed properties of $\zeta$ Pup. We performed over 1 million single star-binary and binary-binary scattering experiments. The details of these experiments are given elsewhere (Belkus et al., 2008). We explored the effects of different masses and different binary periods and eccentricities and, obviously, many experiments reproduce $\zeta$ Pup, but to obtain a runaway velocity as observed the binaries participating in the scattering process always have to be very close (periods smaller than 100 days). Most interestingly, in all our experiments, $\zeta$ Pup turns out to be a merger of 2 or 3 stars.

\section{Conclusions}

In the present paper we first discuss the dynamical formation (due to runaway merging) and evolution of very massive stars (with masses up to $1000 M_{\odot}$ and more) in the cores of young dense clusters. To predict whether such a very massive object becomes a stellar mass black hole, an intermediate mass black hole or explodes as a pair instability supernova, one has to combine a dynamical N-body code with a massive and very massive star evolutionary library, that considers in detail the importance of stellar winds and of the metallicity dependence of these winds on the core hydrogen burning and core helium burning evolution of the massive and very massive stars. Secondly we present arguments in favour of the dynamical ejection scenario in order to explain the runaways (space velocity larger than $30 \mathrm{~km} / \mathrm{s}$ ) with a mass larger than $40 M_{\odot}$, like $\zeta$ Pup, $\lambda$ Cep and $\mathrm{BD}+43^{\circ} 3654$. We conclude:

1. In clusters with solar or supersolar metallicity, black holes form with a mass smaller than 70-75 $M_{\odot}$, but the formation of an intermediate mass black hole with a mass of several $100 M_{\odot}$ is less likely.

2. Due to the metallicity dependence of the stellar wind mass loss, the occurence of pair instability supernovae or the formation of an intermediate mass black hole in dense clusters becomes more probable for smaller metallicities.

3. It is plausible that at least some of the most massive runaways, like $\zeta$ Pup, $\lambda$ Cep and $\mathrm{BD}+43^{\circ} 3654$, are formed during a dynamical encounter of a massive single star and a massive close binary, or by two massive close binaries. In this case, the runaway is the merger of 2 or 3 massive stars.

\section{Ackowledgement}

We thank Dr. Lev Yungelson who calculated the evolution of the $500 M_{\odot}$ star with his version of the Eggleton code but with our preferred stellar wind mass loss formalism. 


\section{References}

Arnault, P., Kunth, D., \& Schild, H. 1989, A \& A, 224, 73

Belczynski, K., Kalogera, V., \& Bulik, T. 2002, Ap. J., 572, 407

Belkus, H., Van Bever, J., \& Vanbeveren, D. 2007, Ap. J. 659,1576

Belkus, H., Van Bever, J., \& Vanbeveren, D. 2008, in preparation

Blaauw, A. 1961, Bull. Astron. Inst. Netherlands, 15, 265

Cervino, M., \& Mas-Hesse, J. M. 1994, A \& A, 284, 749

Colbert, E. J. M., \& Mushotzky, R. F. 1999, Ap. J., 519, 89

Comeron, F., \& Pasquali, A. 2007, A \& A, 467, L23

Dalton, W. W., \& Sarazin, C. L. 1995, Ap.J., 448, 369

de Jager, C., Nieuwenhuijzen, H., \& van der Hucht, K. A. 1988, A \& A Suppl. Ser., 72, 259

Ebisuzaki, T., et al., 2001, Ap. J., 562, L19

Eldridge, J. J., \& Vink, J. S. 2006, A \& A, 452, 295

Fabbiano, G. 1989, Annual review of astronomy and astrophysics. Volume 27, 87

Figer, D. F., McLean, I. S., \& Morris, M. 1999, Ap. J., 514, 202

Figer, D.F. et al., 2002, Ap. J., 581, 258

Fregeau, J.M. et al., 2004, MNRAS, 352, 1

Freitag, M., Rasio, F. A., \& Baumgardt, H. 2006, MNRAS, 368,141

Ghez, A. M., Klein, B. L., Morris, M., \& Becklin, E. E. 1998, Ap. J., 509, 678

Ghez, A. M., et al., 2000, Nature, 407, 349

Gies, D. R., \& Bolton, C. T. 1986, Ap. J., 304, 371

Gürkan, M. A., Freitag, M., \& Rasio, F. A. 2004, Ap. J., 604,632

Heger, A., \& Woosley, S. E. 2002, Ap. J., 567, 532

Hoogerwerf, R., de Bruijne, J. H. J., \& de Zeeuw, P. T. 2001, A \& A, 365, 49

Leitherer, C., et al., 1999, Ap. J. Suppl. Ser., 123, 3

Leonard, P. J. T., \& Duncan, M. J. 1988, Astron. J., 96, 222

Leonard, P. J. T., \& Duncan, M. J. 1990, Astron. J., 99, 608

Lu, J. R., et al., 2005, Ap. J., 625, L51

Maeder, A. 1991, A \& A, 242, 93

Maillard, J. P., Paumard, T., Stolovy, S. R. \& Rigaut, F. 2004, A \& A, 423, 155

Makino, J. \& Aarseth, S.J., 1992, PASJ 44, 141

Mas-Hesse, J. M., \& Kunth, D. 1991, A \& A Suppl. Ser., 88, 399

McCrady, N., Gilbert, A. M., \& Graham, J. R. 2003, Ap. J., 596, 240

Meynet, G. 1995, A \& A, 298, 767

Meynet, G., \& Maeder, A. 2003, A \& A, 404, 975

Mikkola, S. \& Aarseth, S. J. 1993, Celest. Mech. Dyn. Astron., 57, 439

Perna, R., \& Stella, L. 2004, Ap. J., 615, 222

Pols, O. R., Cote, J., Waters, L. B. F. M., \& Heise, J. 1991, A \& A, 241, 419

Portegies Zwart, S. F., \& McMillan, S. L. W. 2002, Ap. J., 576, 899

Portegies Zwart, S. F., et al., 2004, Nature, 428, 724

Portegies Zwart, S. F., et al., 2006, Ap. J., 641, 319
Portegies Zwart, S. F., Makino, J., McMillan, S. L. W., \& Hut, P. 1999, A \& A, 348, 117

Poveda, A., Ruiz, J., \& Allen, C. 1967, Bol. Obs. Tonantzintla y Tacubaya, 4, 86

Ptak, A., \& Colbert, E. 2004, Ap. J., 606, 291

Schaerer, D., \& Vacca, W. D. 1998, Ap. J., 497, 618

Soria, R. 2007, Astrophys. Space Sci., 311, 213

Suzuki, T.K., et al., 2007, Ap. J., 668, 435

Van Bever, J., Belkus, H., \& Vanbeveren, D., 2008, in preparation

Van Bever, J., \& Vanbeveren, D. 1997, A \& A, 322, 116

Van Bever, J., \& Vanbeveren, D. 2000, A \& A, 358, 462

Van Bever, J., \& Vanbeveren, D. 2003, A \& A, 400, 63

Vanbeveren, D., 1991, A \& A 252, 159

Vanbeveren, D. 1995, A \& A, 294, 107

Vanbeveren, D., \& De Loore, C. 1994, A \& A, 290, 129

Vanbeveren, D., et al., 1998a, New Astron., 3, 443

Vanbeveren, D., Van Bever, J., \& Belkus, H. 2007, Ap. J., 662, L107

Vanbeveren, D., Van Bever, J., \& De Donder, E. 1997, A \& A, 317, 487

Vanbeveren, D., De Loore, C., \& Van Rensbergen, W. 1998b, A \& A Rev., 9, 63

Vanbeveren, D., Van Rensbergen, W., \& De Loore, C. 1998c, The Brightest Binaries, Boston: Kluwer Academic 